\title{
Synergy ED: A new electron diffractometer for microED
}

\section{Fraser White ${ }^{1}$, Akihito Yamano ${ }^{1}$, Sho Ito ${ }^{1}$, Takashi Matsumoto ${ }^{1}$, Hiroyasu Sato ${ }^{1}$, Joseph Ferrara ${ }^{2}$, Mathias Meyer ${ }^{3}$, Michał Jasnowski ${ }^{3}$, Eiji Okunishi ${ }^{4}$ and Yoshitaka Aoyama ${ }^{4}$}

\author{
${ }^{1}$ Rigaku Corporation, Haijima, Tokyo, Japan \\ ${ }^{2}$ Rigaku Americas Corporation, The Woodlands, Texas, USA \\ ${ }^{3}$ Rigaku Polska, Wroctaw, Poland \\ ${ }^{4}$ JEOL Ltd., Akishima, Tokyo, Japan \\ fraser.white@rigaku.com
}

The study of the structure of single crystals has typically been achieved with X-ray diffraction while many decades of progress and research have led to hardware improvements which have pushed the limits of X-ray diffraction. The current generation of home lab instruments allow the study of crystals down to about 1 micron in size with sources such as the FR-X, a high-power rotating anode ${ }^{1}$.

In the quest to study even smaller samples than this, microED has become increasingly popular in recent years ${ }^{2}$. As electrons interact more strongly with a crystalline sample than X-rays do, the study of samples smaller than 1 micron becomes possible and, in fact, necessary. We would like to introduce our solution for microED, the Synergy ED, along with results we have obtained using it, and efforts we have made to improve the quality of results.

[1] Matsumoto, T., Yamano, A., Sato, T. et al. "What is This?" A Structure Analysis Tool for Rapid and Automated Solution of Small Molecule Structures. J Chem Crystallogr (2020).

[2] Nannenga, B.L., MicroED methodology and development. Struct Dyn. (2020) 7(1).

[3] Gruene, T. et al Rapid Structure Determination of Microcrystalline Molecular Compounds Using Electron Diffraction Angew. Chem. Int. Ed. (2018) 57(50): 16313-16317

Keywords: Electron Diffraction, Electron Crystallography, Instrumentation 